Revue internationale de l'économie sociale

Recma

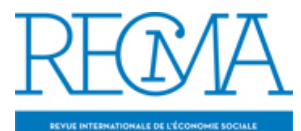

\title{
Évolutions multiples et complexes de l'économie sociale
}

\section{Jean-François Draperi}

Numéro 286, novembre 2002

URI : https://id.erudit.org/iderudit/1022232ar

DOI : https://doi.org/10.7202/1022232ar

Aller au sommaire du numéro

Éditeur(s)

Institut de l'économie sociale (IES)

ISSN

1626-1682 (imprimé)

2261-2599 (numérique)

Découvrir la revue

Citer ce document

Draperi, J.-F. (2002). Évolutions multiples et complexes de l'économie sociale.

Revue internationale de l'économie sociale, (286), 4-4.

https://doi.org/10.7202/1022232ar d'utilisation que vous pouvez consulter en ligne. 


\section{ÉVOLUTIONS MULTIPLES ET COMPLEXES DE L'ÉCONOMIE SOCIALE}

$\mathrm{L}$

'objet de notre revue est d'étudierl'économiesociale, d'analyser ses relationsavecl'Etat, avec les autres économies, dont l'économie dominante, avec les populations, avec ses propres membres, avec elle-même et sa propre histoire. En ce début de Xxi siècle, l'économie sociale vit de multiples évolutions qu'il serait sans doute bien périlleux de tenter de réduire a priori en un ensemble cohérent.

Ce numéro 286 témoigne de la multiplicité et de la complexité des mouvements qui touchent l'économie sociale. C'est si vrai que l'on est en droit de s'interroger, à la suite de François Espagne, sur l'unité elle-même de l'économie sociale et solidaire.

M. Vézina et J.-P. Girard étudient les transferts de compétences et de prestations de l'Etat vers les coopératives au Canada, transfert instituant à la fois des processus de sous-traitance et une alternative au service public.

M.-C. Malo et A. Lapoutte analysent le partenariat entre une banque coopérative (ex-publique) et l'ADIE (France) au service de personnes éprouvant des difficultés à accéder au crédit.

C. Perrin se penche sur une question inexplorée, celle des relations entre un mouvement coopératif (les coopératives artisanales) et le gouvernement de Vichy durant la Seconde Guerre mondiale en France. On observe cette fois un Etat doté d'une volonté conquérante... et dont les réalisations sont finalement mineures.

F. Rousseau décrypte la double mission de gestion et de militantisme que sont dans l'obligation de réaliser les responsables d'associations éducatives reconnues par les pouvoirs publics, ceux-ci devenant en quelque sorte les médiateurs de pratiques entrepreneuriales et gestionnaires dans l'espace associatif.

F. Zandonai décrit la consolidation des coopératives sociales italiennes : leur succès grandissant sur l'ensemble du territoire et leur réunion en réseaux témoignent auprès de ceux qui pouvaient encore en douter que les nouvelles formes d'associations et de coopératives constituent non pas seulement un espace d'expérimentation, mais également un véritable mouvement socio-économique. Cette consolidation ne se fait pas - là comme ailleurs - sans montrer des visages contradictoires de la coopération, dégageant tantôt un profil « adaptatif ", tantôt un profil " proactif ». On lira également avec profit, dans la rubrique "Actualité ", les commentaires aigus de D. Demoustier sur le texte produit par le Medef en juin dernier, dont une partie concerne l'économie sociale. On y trouvera des arguments contestant les positions du mouvement des patrons de l'économie capitaliste, positions dont l'apparente fermeté n'a d'égale que la réelle faiblesse conceptuelle.

Enfin, nous ouvrons une nouvelle rubrique, «Pratiques de l'économie sociale », dont l'objet est de présenter des expériences remarquables et des études résolument empiriques centrées sur des entreprises d'économie sociale. Nous l'inaugurons avec une association qui s'est donné pour mission de dévoiler les pratiques discriminatoires au travail. Celles et ceux qui se retrouvent dans la démarche et dans l'objet de cette rubrique sont invités à s'y associer en s'adressant à la rédaction. 